\title{
Histamine and deep brain stimulation: the pharmacology of regularizing a brain
}

\author{
Timothy C. Whalen ${ }^{1}$ and Aryn H. Gittis ${ }^{1,2}$ \\ 'Center for the Neural Basis of Cognition and 'Department of Biological Sciences, Carnegie Mellon University, Pittsburgh, Pennsylvania, USA.
}

\begin{abstract}
Parkinson's disease (PD) patients have increased histamine in their basal ganglia, but the role of this neurotransmitter in PD is poorly understood. In this issue of the $J C l$, Zhuang et al. demonstrate that histamine levels rise in the subthalamic nucleus (STN) to compensate for abnormal firing patterns. Injection of histamine into the STN restores normal firing patterns and motor activity, whereas merely changing firing rates has no behavioral effect. Moreover, STN deep brain stimulation, a widespread therapy for PD, regularizes firing through endogenous histamine release. This suggests that abnormal firing patterns, rather than rates, cause PD symptoms, and this histaminergic pathway may lead to new treatments for the disease.
\end{abstract}

\section{What defines the Parkinsonian brain?}

Parkinson's disease (PD) afflicts over 10 million people worldwide (1) with a host of severe motor symptoms. A root cause for this dysfunction is the death of dopamine-releasing neurons that innervate a set of deep brain regions called the basal ganglia (BG). However, identification of the process that leads from dopaminergic cell death to motor symptoms has remained contested for decades. How exactly does the loss of BG dopamine produce such profound motor deficits?

Broadly, two camps have formed to answer this question. One explanation, the firing-rate hypothesis, suggests that dysregulation of firing rates across BG nuclei sends excessive "stop" signals, and reduces "go" signals, to the brain's movement center in the motor cortex. The subthalamic nucleus (STN), for instance, shows a markedly increased firing rate in PD. Some suggest that deep brain stimulation (DBS) of the STN, a common treatment for advanced PD, might essentially silence the signals sent by the STN (2), thereby fixing the symptoms caused by STN hyperactivity.
A second explanation, the firing pattern hypothesis, suggests that symptoms arise not from a change in firing rate, but rather a change in the pattern of the activity generated in the BG. Animal models of PD show that firing patterns change significantly after loss of dopamine. For instance, neurons in the STN lose their typical pacemaking pattern in exchange for irregular, bursting, or oscillatory activity. DBS in the STN weakens these unusual patterns in BG output nuclei and the motor cortex (2), though how DBS achieves this, and whether this is responsible for the cessation of Parkinsonian symptoms, is unclear.

Understanding which, if any, of these features causes the motor symptoms of PD could suggest better ways to effectively treat the disease, but how can we disentangle such features of neural communication? In the current study published in the JCI, Zhuang et al. attempt to settle this dispute (3). In doing so, they demonstrate how an unlikely neurotransmitter - histamine - may underlie not only the brain's own attempts to fix pathological firing patterns, but also the mystery behind DBS's success.

Related Article: p. 5413

Conflict of interest: The authors have declared that no conflict of interest exists.

Reference information: J Clin Invest. 2018;128(12):5201-5202. https://doi.org/10.1172/JCI124777.

Regularizing the brain: a novel role for histamine

The neurotransmitter histamine has been found in abundance in the BG of PD patients, but its relevance to the disease has remained unclear. Zhuang et al. showed, surprisingly, that an injection of histamine directly into the STN of Parkinsonian rats lessened their motor symptoms. This injection both regularized and further increased the firing rate of STN neurons (noting that, in PD, firing rates in the STN are already increased above healthy controls). To dissect which of these manipulations led to the behavioral effects, they used a solution containing a high concentration of $\mathrm{K}^{+}$ions, known to increase STN firing rates without affecting spiking patterns. Unlike with histamine, they showed that this solution did not ameliorate the rats' symptoms, suggesting that the pattern changes due to histamine are critical for its effects.

How does histamine cause these changes? The authors demonstrated that this effect is mediated by a subtype of hyperpolarization-activated cyclic nucleotide-gated (HCN) channels, an ion channel present in STN neuronal membranes. HCN channel activation regularizes firing patterns in other BG regions, and these channels are downregulated in PD models (4). However, this interaction between $\mathrm{HCN}$ channels and histamine is new - specifically, the authors show that histamine interacts with $\mathrm{H} 2$-type histamine receptors coupled to HCN channels to increase ion flow through them, directly leading to regularization of firing patterns (Figure 1). Drugs that target these $\mathrm{H} 2$ receptors or HCN channels are sufficient to cause the same effects as histamine or mask histamine's actions, and they have the expected effects on PD symptoms, as predicted by the model in Figure 1, solidifying their role in the regularization process.

Not only does the brain use this pathway to combat abnormal firing patterns in PD, it may be precisely how our current therapies treat the disease. DBS, despite decades of success with patients, remains mysterious 
A

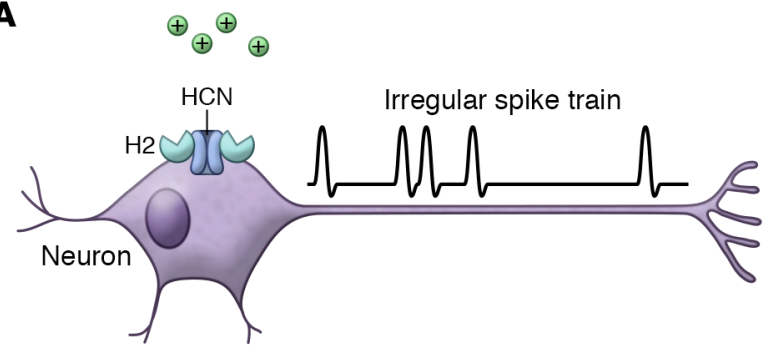

B

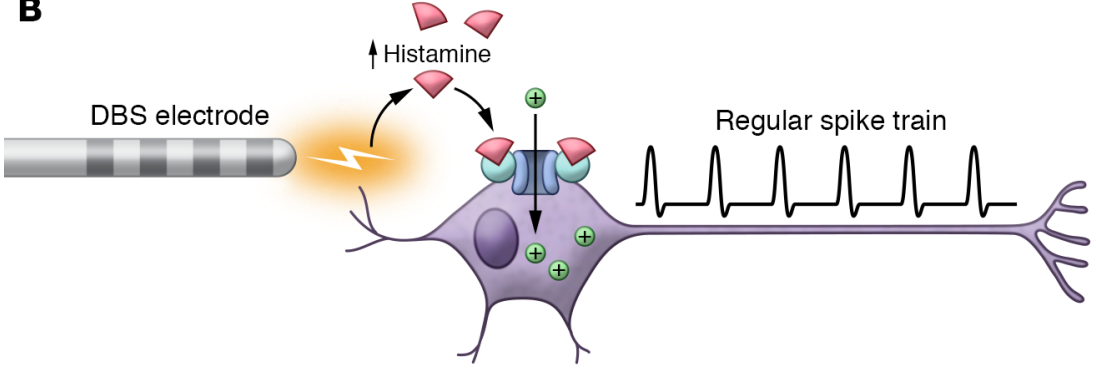

in its therapeutic mechanisms. Zhuang et al. show that DBS in the STN unexpectedly causes the release of endogenous histamine. Blocking the pathway indicated in Figure 1 by downregulating HCN2 channels significantly decreases DBS's ability to ameliorate motor symptoms and regularize BG firing patterns, suggesting that this histamine-led pathway is critical for successful DBS therapy in PD. Knowing this, interventions that better, or more directly, lead to histamine release in the STN may be candidates for new PD treatments.

\section{Causal, compensatory, or neither?}

This study gets at the heart of a question that underlies disease research - for any change observed in a disease state, is it causing or caused by the underlying disorder? And if it is caused by the disease, is it an attempt to correct the dysfunction or merely an unimportant side effect of disease progression?

Excess histamine has been found in the BG of PD patients, leading some to suggest a causal link between histamine and disease progression $(5,6)$. Zhuang and colleagues' work suggests otherwise - that increased histamine is a compensatory mechanism in PD. Many questions remain, however - while this study shows that increased histamine arrives from new fibers from the tuberomammillary nucleus, the only known source of histamine in the brain, it is unclear what leads to this new innervation or how DBS causes histamine release. It also is still possible that, despite histamine's positive effect on symptom relief, this attempt at compensation may contribute to further neurodegeneration through an unknown mechanism.

HCN channels have also previously been implicated in PD, and their downregulation in PD has been shown to directly cause the loss of neural pacemaking in another BG nucleus, the globus pallidus externa (GPe). However, while overexpression of HCN channels in GPe restores pacemaking, it fails to fix PD symptoms (4), suggesting that neither HCN channels nor firing pattern changes are responsible for motor symptoms in PD. On the other hand, HCN channels in the STN have been sparsely studied until now. The present study may indicate that HCN channels and abnormal firing patterns in the STN, rather than GPe, are what must be targeted for symptom reversal. Alternatively, activation of HCN channels in multiple regions, not just the STN, could be necessary for the therapeutic effects seen in this study.

While Zhuang et al. focus primarily on whether cells fire regularly or irregularly, it is important to consider which forms of irregularity may generate PD symptoms. Bursts of spikes, oscillations in firing rate, and Poisson-like spiking with no clear dependence on spiking history are distinct neural patterns that can be mixed and matched in a single neuron (7). For instance, excessive $\beta$ band (13-30 Hz) oscillations arise in PD patients, but it is unclear whether they are causal (8) or merely correlative (9). Oscillations at lower frequencies, while present in
Figure 1. A model of Zhuang et al.'s proposed STN regularization mechanism. (A) An STN neuron in a Parkinsonian brain fires an irregular spike train due to downregulation of HCN channels. (B) DBS evokes endogenous histamine release. Histamine binds to $\mathrm{H} 2$ receptors coupled to HCN channels, which allows them to open, thereby regularizing the STN neuron's spike train.

PD (10), are much less studied. The reduction of one or more of these patterns may be necessary to treat Parkinsonian symptoms, while other pattern changes could be irrelevant side effects of disease progression. A thorough analysis of each of these factors will be necessary to better understand how motor commands become scrambled in the Parkinsonian brain.

Address correspondence to: Aryn H. Gittis, Biological Sciences and Center for the Neural Basis of Cognition, Carnegie Mellon University, 4400 Fifth Avenue, Pittsburgh, Pennsylvania 15217, USA. Phone: 412.268.7229; Email: agittis@cmu.edu.

1. Parkinson's Foundation. Understanding Parkinson's: Statistics. PF website. http://parkinson. org/Understanding-Parkinsons/Causesand-Statistics/Statistics. Updated October 13, 2017. Accessed October 9, 2018.

2. Montgomery EB, Gale JT. Mechanisms of action of deep brain stimulation(DBS). Neurosci Biobehav Rev. 2008;32(3):388-407.

3. Zhuang QX, et al. Regularizing firing patterns of rat subthalamic neurons ameliorates parkinsonian motor deficits. JClin Invest. 2018;128(12):5413-5427.

4. Chan CS, et al. HCN channelopathy in external globus pallidus neurons in models of Parkinson's disease. Nat Neurosci. 2011;14(1):85-92.

5 . Rinne JO, et al. Increased brain histamine levels in Parkinson's disease but not in multiple system atrophy. J Neurochem. 2002;81(5):954-960.

6. Shan L, et al. Alterations in the histaminergic system in the substantia nigra and striatum of Parkinson's patients: a postmortem study. Neurobiol Aging. 2012;33(7):1488.e1-1488.e13.

7. Kaneoke Y, Vitek JL. Burst and oscillation as disparate neuronal properties. J Neurosci Methods. 1996;68(2):211-223.

8. Little S, Brown P. The functional role of beta oscillations in Parkinson's disease. Parkinsonism Relat Disord. 2014;20(suppl 1):S44-S48.

9. Eusebio A, Brown P. Synchronisation in the beta frequency-band - the bad boy of parkinsonism or an innocent bystander? Exp Neurol. 2009;217(1):1-3.

10. Steigerwald F, et al. Neuronal activity of the human subthalamic nucleus in the parkinsonian and nonparkinsonian state. J Neurophysiol. 2008;100(5):2515-2524. 